\title{
Effect of Left and Right Nostril Breathing on Heart Rate Variability among Healthy Adult Males: A Cross-sectional Study
}

\author{
D. Ashwini ${ }^{1}$, V. Anandraj ${ }^{2}$ \\ ${ }^{1}$ Assistant Professor, ${ }^{2}$ Junior Resident, Dept. of Paediatrics, Govt. Villupuram Medical College, Villupuram
}

\begin{abstract}
The effects of various breathing manoeuvres on autonomic functions, especially those of alternate nostril breathing and single nostril breathing have been well documented. ${ }^{1,2,3,4}$ However, most of the studies have investigated these effects after practiced breathing manoeuvres, ${ }^{5,6,7}$ and few have looked into the immediate effects of such types of breathing. The purpose of this study was to determine the immediate effects of a single session of single nostril breathing, first right nostril and then left nostril, on the various time and frequency domain parameters of heart rate variability (HRV) and to compare these effects with that of HRV during normal breathing.
\end{abstract}

Sixty male volunteers (age range, 20 to 40 years) were assessed. Electrocardiography was conducted, using standard procedure, under controlled laboratory conditions, for three five-minute periods: during breathing normally through both nostrils, during the last five minutes of a twenty-minute session of breathing through the right nostril only and similarly while breathing through the left nostril only. The R-R intervals obtained were analyzed for HRV parameters and further analyzed using different statistical method.

When compared to right nostril breathing, a statistically significant increase in mean R-R intervals was noted with left nostril breathing $(\mathrm{p}=0.009)$. Sympathetic activation with right nostril breathing was also evident, although statistically not found to be significant. A statistically significant higher SDNN with left nostril breathing as compared to both nostril breathing $(p=0.004)$ was also observed. Similarly, NN50 and pNN50 were found to be significantly higher with left nostril breathing as compared to both nostril breathing $(\mathrm{p}=$ $0.003 \& 0.007$, respectively). However, RMSSD, LF power, HF power, LF norm, HF norm and LF/HF ratio were not found to be statistically different for the three breathing manoeuvres.

Thus our study showed an overall parasympathetic predominance, with an increase in total power of HRV with left nostril breathing, but the minute variations of HRV were not found to be significantly different among the three breathing manoeuvres.

Keywords: Left nostril breathing Heart rate variability Parasympathetic.

\section{Introduction}

Aim: To compare the effect of right and left nostril

\author{
Corresponding Author: \\ Dr. V. Anandraj, M.D. \\ Junior Resident, Dept. of Paediatrics, Govt. Villupuram \\ Medical College, Villupuram \\ e-mail: visanand83@gmail.com
}

breathing among the healthy adult male volunteers on heart rate variability.

\section{Material and Method}

This cross-sectional descriptive study was conducted in the Department of Physiology, P.I.M.S., between January and December 2012. Ethical clearance was obtained. The study participants were clinically healthy adult male volunteers in the age group of 20-40 years. The exclusion criteria were as follows: 
- Smokers and alcoholics

- Practitioners of yoga

- Participants with any mechanical or infective nasal blockage

- Participants with oro-pharyngeal infections

- Participants on any ANS modifying drugs

- Participants with any major systemic disorders, especially respiratory and cardiovascular disorders

\section{Methodology}

Sixty adult males in the age group of 20-40 years attended the study voluntarily. Prior to being recruited for the study, the participants were briefed about the procedure and written informed consent was obtained from each of them.

In the lab, after preliminary briefing, the participant was asked to lie down in the supine position and breathe in a relaxed manner, without going off to sleep. After a 10 min rest, a 5 min ECG was recorded. This was the first recording for each participant, henceforth designated as $\mathrm{BN}$ (to indicate breathing through both nostrils).

Subsequently, the subject was instructed to occlude his left nostril (with his left index finger) and breathe only through his right nostril for the next 20 minutes. Instruction was also given to the participant not to breathe through the mouth. The investigator monitored and ensured that the participants performed this manoeuvre correctly. During the last 5 minutes of this right nostril breathing manoeuvre ECG was recorded as with the $\mathrm{BN}$ recording. This was the second recording for each participant, henceforth designated as RN (to indicate right nostril breathing)
The participant was then asked to breathe in a relaxed manner through both nostrils, while resting in the supine position for a period of 10 minutes. Following this period of rest, the participant was asked to occlude his right nostril (with his right index finger) and breathe only through his left nostril for the next 20 minutes. As with the RN manoeuvre, ECG was recorded during the last 5 minutes of this left nostril breathing manoeuvre. This was the third recording for each participant, henceforth designated as LN (to indicate left nostril breathing).

Equipment and Data Acquisition: For the purpose of ECG recording, the three standard limb leads were used, with $3 \mathrm{M}$ monitoring electrodes placed over the anterior chest wall. The electrodes were connected to a computerized ambulatory ECG system (Niviquire, Pune). The ECG signal was continuously amplified, digitized and stored in the computer for offline analysis. The RR peak detector was adjusted appropriately. A high quality ECG recording was thus acquired under standardized conditions to minimise artefacts. R-R intervals acquired from this ECG monitoring system were then further subjected to HRV analysis.

For the purpose of HRV analysis, Kubios HRV version 2.0, 2008 (Biosignal Analysis and Medical Imaging Group, University of Kuopio, Finland) was used. The R-R interval data obtained with the ECG monitoring system was subjected to Fast Fourier Transformation and analyzed for parameters in the time and frequency domains.

Statistical Analysis: The data was entered in Microsoft Excel and analyzed using SPSS 17.0 for Windows statistical software. Repeated measures ANOVA and students paired t-test were used.

\section{Result}

Table 1: Mean Standard Deviation of NN Intervals (SDNN) with the Three Breathing Manoeuvres (n=60)

\begin{tabular}{|l|c|c|c|c|}
\hline \multirow{2}{*}{} & \multirow{2}{*}{ Median (ms) } & \multicolumn{2}{|c|}{ Interquartile Range } & \multirow{2}{*}{ p value* } \\
\cline { 3 - 4 } & & $\mathbf{2 5}^{\text {th }}$ Percentile & $\mathbf{7 5}^{\text {th }}$ Percentile & \\
\hline Both nostril breathing (BN) & 46.05 & 33.63 & 88.05 & \multirow{2}{*}{0.001} \\
\hline Right nostril breathing (RN) & 46.50 & 37.70 & 87.28 & 92.45 \\
\hline Left nostril breathing (LN) & 62.60 & 46.13 & \\
\hline
\end{tabular}

*Friedman test used; statistically significant at $\mathrm{p}=0.05$ level 
Table 2: Root Mean Square of Successive Deviations (RMSSD) with the Three Breathing Manoeuvres $(\mathrm{n}=60)$

\begin{tabular}{|l|c|c|c|c|}
\hline \multirow{2}{*}{} & \multirow{2}{*}{ Median (ms) } & \multicolumn{2}{|c|}{ Interquartile Range } & \multirow{2}{*}{ p value* } \\
\cline { 3 - 4 } & & $\mathbf{2 5}^{\text {th }}$ Percentile & $\mathbf{7 5}^{\text {th }}$ Percentile & \\
\hline Both nostril breathing (BN) & 36.50 & 25.88 & 85.78 \\
\hline Right nostril breathing (RN) & 34.90 & 27.85 & 74.03 & \multirow{2}{*}{0.059} \\
\cline { 1 - 4 } Left nostril breathing (LN) & 46.30 & 31.33 & 81.68 \\
\hline
\end{tabular}

*Friedman test used; statistically significant at $\mathrm{p}=0.05$ level

Table 3: Median NN50 Values with the Three Breathing Manoeuvres $(n=60)$

\begin{tabular}{|l|c|c|c|c|}
\hline \multirow{2}{*}{} & \multirow{2}{*}{ Median } & \multicolumn{2}{|c|}{ Interquartile Range } & \multirow{2}{*}{ p value* } \\
\cline { 3 - 4 } & & $\mathbf{2 5}^{\text {th }}$ Percentile & $\mathbf{7 5}^{\text {th }}$ Percentile & \\
\hline Both nostril breathing $(\mathrm{BN})$ & 24.50 & 12.25 & 63.75 & \multirow{2}{*}{0.009} \\
\hline Right nostril breathing $(\mathrm{RN})$ & 30.50 & 13.25 & 84.50 & \\
\hline Left nostril breathing $(\mathrm{LN})$ & 52.00 & 18.25 & 94.50 & \\
\hline
\end{tabular}

*Friedman test used; statistically significant at $\mathrm{p}=0.05$ level

Table 4: Median pNN50 Values with the Three Breathing Manoeuvres $(n=60)$

\begin{tabular}{|l|c|c|c|c|}
\hline \multirow{2}{*}{} & \multirow{2}{*}{ Median (\%) } & \multicolumn{2}{|c|}{ Interquartile Range } & \multirow{2}{*}{ p value* } \\
\cline { 3 - 4 } & & $\mathbf{2 5}^{\text {th }}$ Percentile & $\mathbf{7 5}^{\text {th }}$ Percentile & \\
\hline Both nostril breathing (BN) & 10.40 & 5.20 & 28.08 \\
\hline Right nostril breathing (RN) & 12.00 & 5.20 & 33.10 & \multirow{2}{*}{0.021} \\
\hline Left nostril breathing (LN) & 20.40 & 7.55 & 37.03 \\
\hline
\end{tabular}

*Friedman test used; statistically significant at $\mathrm{p}=0.05$ level

Table 5: Low Frequency Power Values with the Three Breathing Manoeuvres $(n=60)$

\begin{tabular}{|l|c|c|c|c|}
\hline \multirow{2}{*}{} & \multirow{2}{*}{ Median $\left(\mathbf{m s}^{\mathbf{2}}\right)$} & \multicolumn{2}{|c|}{ Interquartile Range } & \multirow{2}{*}{ p value* } \\
\cline { 3 - 4 } & & $\mathbf{2 5}^{\text {th }}$ Percentile & $\mathbf{7 5}^{\text {th }}$ Percentile & \\
\hline Both nostril breathing (BN) & 836.50 & 327.50 & 1925.25 \\
\hline Right nostril breathing (RN) & 664.50 & 319.25 & 2282.50 & \multirow{2}{*}{0.016} \\
\hline Left nostril breathing (LN) & 1049 & 651.75 & 2085 & \\
\hline
\end{tabular}

*Friedman test used.

\section{Discussion}

The participants in our study had a mean age of 29.85 \pm 6.22 years (range 20-40 years). Their mean height, weight and BMI were $167.7 \pm 6.45 \mathrm{~cm}, 66.52 \pm 9.57 \mathrm{~kg}$ and $23.58 \pm 2.66 \mathrm{~kg} / \mathrm{m}^{2}$ respectively. Their baseline vital parameters measured showed the mean pulse rate to be $77.88 \pm 6.71 \mathrm{bpm}$, the mean systolic blood pressure to be $117.80 \pm 9.72 \mathrm{mmHg}$ and the mean diastolic blood pressure to be $77.37 \pm 5.12 \mathrm{mmHg}$.
Time domain parameters: A reduction in heart rate after left nostril breathing has been reported, ${ }^{8}$ while others have reported no significant changes. ${ }^{3,4}$ Dane et al, found an increase in heart with both nostril breathing, due to overall effect of exercise on heart rate. ${ }^{9}$

In our study, the highest median SDNN value was observed with left nostril breathing $(62.60 \mathrm{~ms})$ and least with normal breathing $(46.05 \mathrm{~ms})$. When SDNN values were compared, there was a statistically significant 
difference between normal and left nostril breathing. Since SDNN correlates with total power of HRV, it can be concluded from our results that total power of HRV was increased with left nostril breathing, significantly, as compared to both nostril breathing. That the SDNN of right nostril breathing was almost similar to that of both nostril breathing $(46.50 \mathrm{~ms})$ shows a balancing effect of right nostril breathing.

In our study, the highest median value for NN50 was observed with left nostril breathing (52) and the lowest median value was observed with normal breathing (24.50). When the median NN50 values were compared for the three breathing manoeuvres, there was a statistically significant difference observed between both nostril and left nostril breathing.

Similarly, the highest median value for pNN50 was observed with left nostril breathing (20.40\%) and the lowest median value was observed with normal breathing $(10.40 \%)$. When the median pNN50 values were compared, statistically significant difference was observed with normal and left nostril breathing.

Both NN50 and pNN50 correlate well with the HF power of the frequency domain analysis and are indicative of parasympathetic activity. Thus, these results indicate an increase of parasympathetic activity with left nostril breathing as compared to both nostril breathing.

However, RMSSD, which is considered to be a better indicator of the short-term component of HRV, signifying parasympathetic activity, was not found to be significantly different among the three breathing manoeuvres. Also there were no differences between right nostril breathing and both or left nostril breathing in terms of RMSSD, NN50 or pNN50 shows a balancing effect of right nostril breathing with both sympathetic and parasympathetic effects on the heart.

Frequency domain parameters: A study by Raghuraj et al reported the effects of rapid breathing techniques and slow and deep breathing techniques in 12 healthy male volunteers. They reported that following the slow and deep breathing technique, it was found that there was no significant change, though a non-significant trend towards increase in HF power and LF power was noted. ${ }^{10}$

An unpublished report by Rajajeyakumar et al has shown increased LF power and decreased HF power with right nostril breathing. ${ }^{11}$ The index of sympathovagal balance as reflected by $\mathrm{LF} / \mathrm{HF}$ ratio increased i.e. from 1.8 to 2.2 after the intervention. All the observation showed that practiced right nostril breathing is sympathomimetic. In practiced left nostril breathing, the time domain analysis of HRV revealed a decreased heart rate and an increased pNN50. The frequency domain analysis revealed an increased $\mathrm{HF}$ power with decreased LF/HF ratio i.e from 2.1 to 1.5. Thus, left nostril breathing was shown to have a definite parasympathetic effect.

In our study, however, both HF and LF power was found to be highest with left nostril breathing and lowest with right nostril breathing, although the differences were statistically not significant. HF and LF power in normalized units were also not found to be statistically different among the three manoeuvres. Neither were the $\mathrm{LF} / \mathrm{HF}$ ratio noted to be significantly different.

Thus the frequency domain analysis of our data seems to indicate a lack of the minute heart rate variations when subjected to spectral analysis, although there was an overall increase in total power, as well an increase in mean R-R interval with left nostril breathing.

Limitations of our study included the small sample size and the inability to conduct the breathing manoeuvres with sufficient time gap in-between. Also it would have been helpful if this study could have documented the airflow of each nostril before the start of the manoeuvres.

\section{Conclusion}

This cross sectional study was conducted with the aim to observe the effects of forced single nostril breathing manouevres on various HRV parameters.

An increase in the total power of HRV, as indicated by a statistically significant higher SDNN with left nostril breathing as compared to both nostril breathing $(p=0.004)$ was also observed in our study. Other time domain parameters like NN50 and pNN50, which correspond to HF power of the frequency domain and therefore indicate parasympathetic activation, were found to be significant higher with left nostril breathing as compared to both nostril breathing ( $p=0.003 \&$ 0.007, respectively). However, RMSSD, a preferred estimate of the short-term component of HRV was not found to be statistically significantly different with the three breathing manoeuvres. Neither were any of the 
frequency domain parameters like LF power, HF power, $\mathrm{LF}(\mathrm{nu}), \mathrm{HF}(\mathrm{nu})$ and LF/HF ratio found to be statistically different among the three breathing manoeuvres.

Thus we would like to conclude that while single nostril breathing manoeuvres have an overall effect of either parasympathetic (left nostril) or, to a lesser extent, sympathetic (right nostril) activation over the control of heart rate, the frequency variations in R-R intervals do not manifest any obvious predominance by any one division of the autonomic nervous system.

As a treatment option, the advantages of such a manoeuvre are obvious in that it is non-invasive and easy to use by the patient. It will require further investigations to see if the heart-rate-reducing effect of left nostril breathing is as effective in situations of physiological tachycardia and whether such manoeuvres can be utilized clinically in situations where a prompt reduction in an elevated heart rate is essential to treatment.

\section{Conflict of Interest: Nil}

\section{Source of Funding: Self}

Ethical Clearance: Institutional ethical committee (Pondicherry Institute of Medical Sciences).

\section{References}

1. Pal G, Velkumary S, Madanmohan. Effect of shortterm practice of breathing exercises on autonomic functions in normal human volunteers. Indian $\mathrm{J}$ Med Res. 2004;120(2):115-21.

2. Jain N, Srivastava RD, Singhal A. The effects of right and left nostril breathing on cardiorespiratory and autonomic parameters. Indian $\mathrm{J}$ Physiol Pharmacol. 2005;49(4):469-74.

3. Larson G, Zaichkowsky LD, Mostofsky DI. Forced unilateral nostril breathing (FUNB) effects on the autonomic nervous system: an unsupported claim. Med Hypotheses. 1993;41(4):367-9.

4. Shannahoff-Khalsa DS, Kennedy B. The effects of unilateral forced nostril breathing on the heart. Int J Neurosci. 1993;73(1-2):47-60.

5. Udupa KN, Singh RH, Settiwar RM. Studies on the effect of some yogic breathing exercises (pranayama) in normal persons. Indian J Med Res. 1975;63:1062-5.

6. Cooper S, Osborne J, Newton S, Harrison V, Thompson Coon J, Lewis S, Tattersfield A. Effect of two breathing exercises (Buteyko and pranayama) in asthma: a randomised controlled trial. Thorax. 2003;58(8):674-9.

7. Vedanthan $P$ et al. Clinical study of yoga techniques in university students with asthma: a controlled study. Allergy Asthma Proc. 1998;19(1):3-9.

8. Telles S, Nagarathana R, Nagendra HR. Physiological measures of right nostril breathing. J Alt Complement Med. 1996;2(4):479-84.

9. Dane S, Caliskan E, Karasen M, Oztasan N. Effects of unilateral nostril breathing on blood pressure and heart rate in right-handed healthy subjects. Int J Neurosci. 2002; 112(1):97-102.

10. P. Raghuraj, A. G. Ramakrishnan, H. R. Nagendra and Shirley Telles. Effect of two selected yogic breathing techniques on Heart rate variability. Indian J Physiol Pharmacol. 1998;42(4):467-472.

11. Rajajeakumar, Madanmohan, Amudharaj, Bandi Harikrishna, Jeyasettiseloune, Bhavanani, Immediate effect of Suryanadi and Chandranadi Pranayam on Short Term Heart Rate Variability in healthyyoung volunteers. Indian $\mathrm{J}$ Physiol Pharmacol, Vol 55, No.5 supplement. 